\title{
Tabagismo e suas peculiaridades durante a gestação: uma revisão crítica*
}

\author{
Smoking and its peculiarities during pregnancy: a critical review \\ WALDIR LEOPÉRCIO, ANALICE GIGLIOTTI
}

\begin{abstract}
A gestação é uma ocasião especial para a promoção da cessação do tabagismo. A preocupação com a saúde do feto gera uma motivação extraordinária na gestante. Os resultados e a relação custo-efetividade das intervenções são melhores neste grupo do que na população em geral. Os ganhos extrapolam os benefícios à saúde da mulher, pois permitem também o desenvolvimento de um feto mais saudável. 0 conhecimento das peculiaridades do tabagismo durante a gestação é fundamental para uma abordagem direcionada e com maior probabilidade de sucesso. Este trabalho de revisão tem o objetivo de ressaltar a extensão dos malefícios do fumo, tanto para a mulher gestante quanto para seu feto, e estimular o uso de técnicas apropriadas para a suspensão do tabagismo nesta população.
\end{abstract}

J Bras Pneumol 2004; 30(2) 176-185

Descritores: Tabagismo/efeitos adversos. Abandono do uso de tabaco/métodos. Gravidez.

\section{INTRODUÇÃO}

0 tabagismo durante a gestação tem implicações que vão além dos prejuízos à saúde materna. Os malefícios sobre a saúde fetal são tantos, que justificam dizermos que o feto é um verdadeiro fumante ativo.

0 fumo na gravidez é responsável por 20\% dos casos de fetos com baixo peso ao nascer, $8 \%$ dos partos prematuros e 5\% de todas as mortes perinatais ${ }^{1}$. Estudos mostram que o tabagismo na gestação pode contribuir para a síndrome da morte súbita do bebê, além de causar importantes alterações no desenvolvimento do sistema nervoso
Pregnancy is a special occasion to promote the end of the smoking habit. Concern with health of the fetus brings about an extraordinary motivation for the pregnant woman. Results and cost-effectiveness of interventions are better in this group than in the overall population and the advantages extrapolate the benefits to the woman's health as they also allow for the development of a healthy fetus. Knowledge of the peculiarities of smoking during pregnancy is fundamental for a directed approach which adds to a probability of greater success. This review investigates the extent of the harm of tobacco for the pregnant woman as well as for the fetus. As such it, it encourages the use of appropriate techniques to urge pregnant women to stop smoking.

Key words: Smoking/adverse effects. Tobacco use cessation/ methods. Pregnancy.

fetal $^{2}$. Estimativas econômicas indicam que os custos com as complicações perinatais são 66\% maiores nos casos de mães que fumaram durante a gravidez do que nos de mães não fumantes ${ }^{3}$. Embora os maiores benefícios para o desenvolvimento fetal ocorram se a cessação do tabagismo se fizer ainda no início da gestação ${ }^{4}$, a interrupção em qualquer momento da gravidez, ou mesmo no pós-natal, tem significativo impacto na saúde da família. Em torno de $27 \%$ das crianças americanas são expostas ao tabagismo passivo em suas residências ${ }^{5}$ e os custos médicos anuais com os parentes de fumantes são estimados em 4,6 
bilhões de dólares ${ }^{6}$. A manutenção da abstinência no decorrer da gestação e no pós-parto tem papel fundamental na prevenção de doenças maternoinfantis relacionadas ao tabaco.

0 grande ganho à saúde da mãe, do feto e da criança, e a extraordinária motivação materna que a gravidez por si promove justificam a aplicação de esforços especiais para a interrupção do tabagismo na gestação. Inserido neste contexto, o objetivo deste trabalho foi contribuir com uma revisão crítica dos principais artigos nacionais e estrangeiros indexados no MEDLINE e LILACS, dentre outros, publicados até o ano de 2003 sobre o tabagismo e suas peculiaridades durante a gestação.

\section{Toxicidade dos compostos do tabaco na geastante e no feto}

\section{Nicotina}

A insuficiência útero-placentária tem sido indicada como o principal mecanismo responsável pelo retardo do crescimento fetal nas gestantes fumantes. A nicotina causa vasoconstricção dos vasos do útero e da placenta, reduzindo o fluxo sangüineo e a oferta de oxigênio e nutrientes para o feto. No entanto, alguns autores ${ }^{2,7}$ acreditam que além disso, os danos biológicos celulares e moleculares provocados pelo monóxido de carbono e por outras toxinas também têm forte interferência no desenvolvimento do feto.

A exposição pré e perinatal à nicotina tem sido relacionada a alterações da cognição ${ }^{8}$, e do desenvolvimento psico-motor ${ }^{9}$ e sexual no jovem ${ }^{10}$. Estes efeitos parecem ser secundários à neurotoxicidade da nicotina, que interage com os receptores nicotínicos colinérgicos em fase precoce e inadequada durante a gestação, prejudicando a neurogênese e a sinaptogênese ${ }^{11}$.

A sindrome da morte súbita do bebê tem mecanismos causais desconhecidos e provavelmente multifatoriais. 0 fumo durante a gravidez é reconhecidamente um elemento que eleva o risco de sua ocorrência ${ }^{12}$. Uma das hipóteses para explicar essa relação é que a exposição prolongada da medula adrenal do feto à nicotina leva à perda de sua capacidade de responder reflexamente à hipóxia ${ }^{13}$. Sendo assim, durante a apnéia transitória ou obstrução das vias aéreas do recém-nato, não haveria liberação de catecolaminas para redistribuição do fluxo sangüíneo para o cérebro e coração e para a manutenção da freqüência cardíaca durante a hipóxia.

Gilliland et al. mostraram que a exposição fetal aos compostos do tabaco compromete o crescimento dos pulmões e leva à redução das pequenas vias aéreas, implicando em alterações funcionais respiratórias na infância, que persistem ao longo da vida $^{14,15}$. 0 desenvolvimento pulmonar modificado pode estar associado ao aumento do risco futuro de doença pulmonar obstrutiva crônica (DPOC), câncer de pulmão e doenças cardiovasculares ${ }^{16-18}$.

A nicotina provoca alterações súbitas e momentâneas no aparelho cardiovascular da gestante, com elevação da freqüência cardíaca e das pressões arteriais sistólicas e diastólicas. Essas mudanças são dose-dependentes e parecem não provocar repercussão clínica significativa maternofetal ${ }^{19}$.

\section{Monóxido de carbono}

0 Monóxido de carbono (CO) é um gás venenoso produzido pela combustão incompleta de matéria orgânica. Embora existam outras fontes de exposição ao CO, como a poluição atmosférica, o fumo passivo, a produção endógena e a exposição ocupacional, nada se compara ao tabagismo ativo ${ }^{20}$.

O co liga-se à hemoglobina materna e fetal no sítio onde se deveria ligar o oxigênio, com afinidade 200 vezes maior que este ${ }^{21}$. 0 produto dessa ligação é a carboxihemoglobina ( $\mathrm{COHb}$ ), que tem uma meia vida de eliminação de cinco a seis horas $^{22}$, com variação de concentração no sangue dos fumantes de 5\% a 10\%. A hemoglobina fetal tem uma ligação com o CO mais forte que a hemoglobina materna, resultando em níveis de $\mathrm{COHb}$ mais elevados na circulação fetal ${ }^{23}$. As altas concentrações de $\mathrm{COHb}$ provocam hipóxia tecidual, estimulando a eritropoiese e causando uma elevação do hematócrito da gestante fumante e de seu feto ${ }^{24}$. Isto implica em hiperviscosidade sangüinea, aumento do risco de infarto cerebral no neonato ${ }^{25}$, e mau desempenho da placenta ${ }^{26}$.

O CO altera a curva de dissociação da oxihemoglobina, prejudicando a oxigenação dos tecidos ${ }^{23}$. A hipóxia celular crônica é um dos fatores que podem explicar o retardo do crescimento fetal? 
No sistema nervoso do feto, o CO tem ação de uma potente toxina, e pode causar lesões neurológicas temporárias e/ou permanentes ${ }^{27}$. No sistema cardiovascular, provoca elevação da freqüência cardíaca e hipertrofia miocárdica ${ }^{28}$.

\section{Outros efeitos tóxicos dos compostos do cigarro}

É sabido que o tabagismo leva ao comprometimento do sistema imunológico, com diminuição da capacidade fagocitária dos macrófagos e alteração dos níveis de $\lg$ A nas mucosas $^{29}$. Isto pode explicar porque as gestantes fumantes têm maior risco de abortamento ${ }^{30}$. A ruptura prematura das membranas seria subseqüente a rupturas focais ${ }^{31}$, por sua vez secundárias a infecções locais facilitadas pela toxicidade do fumo.

Outra causa importante de ruptura prematura das membranas e abortamento nas fumantes é o fato de haver uma redução de 50\% na concentração de ácido ascórbico no líquido aminiótico em comparação às não-fumantes ${ }^{32}$. A vitamina $\mathrm{C}$, além de ter papel significativo nas defesas imunológicas ${ }^{33}$, é imprescindível na formação do colágeno que compõe a membrana aminiocoriônica ${ }^{34}$. 0 transporte de aminoácidos pela placenta está reduzido nas fumantes ${ }^{35}$, o que interfere na sintese proteica e contribui para o mau desenvolvimento da membrana aminiocoriônica ${ }^{36}$.

Outro fator responsável pelo aumento de abortamentos em fumantes é a redução da síntese placentária de óxido nítrico, um potente relaxante do miométrio ${ }^{37}$.

0 fator de ativação das plaquetas está envolvido no início e na manutenção do trabalho de parto, através da síntese de prostaglandinas. 0 tabagismo reduz a inativação desse fator, podendo provocar contração uterina e parto prematuro ${ }^{37}$.

\section{Validação bioquímica do perfil tabágico da gestante}

A forte pressão social e o reconhecimento dos malefícios do tabagismo sobre a saúde materna e fetal constrangem as gestantes, fazendo com que grande parte delas não preste informações verídicas a respeito de seu hábito tabágico ${ }^{38}$. Daí a importância, principalmente em levantamentos epidemiológicos e na condução de pesquisas científicas, de essas informações serem validadas ${ }^{39}$.
$\mathrm{Na}$ atualidade, questiona-se a utilização indiscriminada da confirmação bioquímica do auto-relato ${ }^{40}$. Sua importância está diretamente relacionada às características da população estudada ${ }^{41}$. Nas situações onde o risco de falsas informações é elevado, como no caso das gestantes, os auto-relatos precisam ser confrontados para que os dados obtidos ganhem credibilidade. Estudos acerca de tabagismo durante a gestação relatam discordância de $28 \%$ a $50 \%$ entre o auto-relato e os testes de cotinina ${ }^{42}$.

No acompanhamento de gestantes, dependendo da informação que se esteja buscando, o meio de validação pode variar. Hoje, os mais utilizados são a dosagem da cotinina (saliva ou urina) e do monóxido de carbono no ar exalado (Coex), cada qual com suas características próprias.

Caso o objetivo seja definir o perfil tabágico da gestante (diferenciação entre fumantes e nãofumantes), a dosagem da cotinina é o método de eleição, apesar de todas as suas dificuldades técnicas. Se o objetivo é ter um método rápido que comprove a alteração no número de cigarros consumidos, aumentar a motivação para mudança de comportamento e avaliar o tabagismo passivo, a mensuração do COex deve ser preferida ${ }^{43,44}$.

\section{Avaliação crítica dos custos do controle do tabagismo durante a gestação}

Os gastos devidos ao tabagismo durante a gestação são parte dos cuidados especiais dispensados não só às gestantes, mas também aos neonatos e à criança no primeiro ano de vida, que sofrem com o hábito de fumar de suas mães ainda na vida intra-uterina e/ou durante o período de amamentação e crescimento.

Nos EUA, os custos com o tratamento das intercorrências obstétricas provenientes do fumo na gravidez atingem cifras de 135 a 167 milhões de dólares por $\mathrm{ano}^{45}$.

Estimativas econômicas indicam que os gastos médicos diretos com as complicações peri-natais das gestantes fumantes são $66 \%$ maiores do que das não-fumantes ${ }^{46}$. 1sto se deve em grande parte às complicações fetais, como o baixo peso ao nascer (BNP) ${ }^{47}$, segundo a Organização Mundial da Saúde (OMS) o fator isolado mais importante de morbimortalidade infantil ${ }^{48}$, a síndrome da morte súbita do bebê $\hat{e}^{12,49}$, e os distúrbios ventilatórios ${ }^{14,15}$, dentre outras ${ }^{49}$. 
Nos países em desenvolvimento, o tabagismo durante a gestação é, ao lado da desnutrição materna e da falta de assistência pré-natal, a principal causa de $\mathrm{BPN}^{47}$. Nos países e centros desenvolvidos é a primeira causa isolada ${ }^{50}$.

Em trabalho publicado na Health Economics ${ }^{51}$, Adams et al. afirmam que o fumo na gestação eleva em 20\% o risco de assistência em UT1 neonatal, e que o tempo de permanência e os gastos com estas crianças na UTI neonatal são maiores do que aqueles cujas mães não fumaram durante a gravidez. Concluem que o tabagismo durante a gestação aumenta em $\$ 700,00$ os custos com a assistência neonatal.

A manutenção do hábito de fumar durante o primeiro ano de vida da criança agrava os prejuízos respiratórios, neurológicos e psico-motores que se iniciaram ainda intra-útero ${ }^{14,52}$, avolumando os gastos com assistência médica dos filhos de fumantes.

Baseado na definição de Doubilet et al. de "custo efetivo" na Medicina ${ }^{53}$, para se determinar a relação custo-benefício de um programa é necessário avaliar o seu impacto sobre a melhoria da saúde e a economia de dinheiro proporcionada, ou observar o resgate dos benefícios de saúde mediante um custo aceitável. Os programas de prevenção e tratamento do tabagismo apresentam a melhor relação custo-benefício dentre todas as estratégias de promoção de saúde, e têm o maior impacto sobre a expectativa de vida do ser humano, se comparados a qualquer outra ação preventiva isolada ${ }^{54}$. 0 Banco Mundial assegura que investir no controle do tabagismo é altamente rentável, sobretudo para os paises em desenvolvimento ${ }^{55}$.

Se considerarmos o controle do tabagismo pontualmente durante a gestação, a relação custobenefício ficará maximizada. Os benefícios gerados pela cessação não se resumem à saúde da gestante, mas também do feto e de sua família. Se a gestante interromper o tabagismo até a $20^{\text {a }}$ semana da gestação, o risco de ter um bebê com BPN iguala-se ao risco das não fumantes ${ }^{56}$. A consciência da gravidez, por si só, já exerce uma motivação adicional na gestante para considerar a cessação. Além disso, as visitas pré-natais proporcionam várias oportunidades de reforço das intervenções para interrupção do fumo, o que eleva a possibilidade de êxito ${ }^{57}$. Este é o momento mais propício para auxiliar uma mulher no abandono do tabagismo. As taxas de sucesso dos programas de intervenção para o abandono do fumo são cerca de três vezes maiores nas gestantes quando comparadas com outros grupos ${ }^{58}$. A precocidade e a facilidade da mensuração do retorno do investimento motiva autoridades e gestores de saúde, uma vez que ao término da gestação os ganhos com a saúde da gestante e do neonato, e a economia deles decorrente, já podem ser contabilizados.

Para cada dólar investido no controle do tabagismo durante a gestação, são recuperados seis nos ganhos peri-natais e até dezessete dólares se estendermos a somatória dos benefícios ao primeiro ano de vida da criança ${ }^{50,59}$. Mesmo assim, em países como os EUA e Canadá, onde políticas governamentais anti-tabágicas são agressivas e são empregados investimentos maciços para se controlar o fumo durante a gestação, muito há para se conquistar. Cerca de 20\% das norteamericanas $^{60}$ e $24 \%$ das canadenses ${ }^{61}$ ainda fumam durante a gestação. Somente 49\% dos ginecoobstetras americanos advertem suas pacientes quanto às conseqüências do tabagismo, e apenas 28\% discutem estratégias de abandono ${ }^{62}$.

Em levantamento realizado em instituições públicas e privadas da Califórnia (EUA) que prestam assistência pré-natal, constatou-se que $8 \%$ não oferecem qualquer tipo de apoio à gestante para cessação do tabagismo ${ }^{63}$. Dentre os planos de cuidado gerenciado (managed care), somente $45 \%$ disponibilizam para as gestantes as recomendações padronizadas da Agency for Health Care Policy and Research ${ }^{64}$.

Apesar de os dados dos EUA não serem exatamente superponíveis aos do Brasil, podemos inferir que a nossa realidade se equivale ou é pior do que a deles. Ou seja, estima-se que o tabagismo entre as gestantes brasileiras também compromete milhares de vidas e consome milhões de reais que poderiam estar sendo empregados, por exemplo, na melhoria da atenção pré-natal e no controle da mortalidade infantil.

\section{Prevenção e tratamento do tabagismo durante a gestação}

A capacitação e o treinamento de profissionais para o controle do tabagismo e a implementação de programas públicos e privados para apoio à 
cessação também revelaram-se extremamente eficazes na redução do tabagismo entre gestantes ${ }^{38,42,65}$.

A prevalência de norte-americanas grávidas fumantes é maior entre as jovens (menores de 20 anos) ${ }^{66}$ e entre aquelas com baixa escolaridade ${ }^{67}$. Estudos com a população brasileira confirmam esses achados ${ }^{68-71}$. Portanto, para se reduzir o tabagismo na gestação, além de se investir em ações de saúde, deve-se também investir em educação e ter as adolescentes como um dos alvos prioritários $^{72}$.

Intervenções mínimas praticadas com a população em geral podem alcançar taxa de sucesso de cessação do tabagismo de até 30\% $0^{73}$. Os resultados desses esforços são ainda superiores quando direcionados especificamente às gestantes ${ }^{58,74}$. A gestação e o pós-parto são um momento ímpar para promoção da cessação do tabagismo ${ }^{75}$. Logo, é importante que em todo acompanhamento pré-natal sejam dispensados alguns minutos sobre o tema tabagismo e suas conseqüências. Na verdade, toda mulher em idade fértil e que esteja considerando a hipótese de engravidar, já deveria ser abordada pelo seu ginecologista, no sentido da prevenção dos efeitos do tabagismo durante a gestação. Essas advertências também devem ser estendidas às gestantes fumantes passivas ${ }^{76}$ e aos seus maridos, já que ter um cônjuge fumante é fator preditivo de insucesso para a cessação do hábito de fumar ${ }^{67}$.

No Brasil existe uma demanda reprimida em relação a vagas para tratamento da dependência da nicotina ${ }^{77}$. Visando atenuar este panorama, em agosto de 2002 foi assinada uma portaria do Ministério da Saúde ${ }^{78}$ que inclui o tratamento contra o fumo nos serviços do sistema público de saúde. No entanto, terapias específicas para gestantes ainda não estão previstas.

A farmacoterapia e o aconselhamento são medidas de eficácia comprovada na cessação do tabagismo. Cada qual tem sua eficácia individual, mas se somadas aumentam as taxas de sucesso ${ }^{79}$. A combinação do aconselhamento com o uso da bupropiona e/ou formas de terapia de reposição de nicotina (TRN) apresenta taxa pontual de cessação de $40 \%$ a $60 \%$, e ao fim de um ano, de $25 \%$ a $30 \%{ }^{79}$. A terapia farmacológica auxiliar na cessação do tabagismo tem uso muito restrito para a gestante. Mediante a possibilidade de toxicidade fetal, o emprego destas drogas tem uma relação risco-benefício incerta. Isto reforça a necessidade de se trabalhar o abandono do tabagismo antes mesmo da concepção.

Dempsey e Benowitz ${ }^{7}$, em um detalhado artigo de revisão sobre o uso de TRN durante a gestação, defendem a idéia de que nos casos onde a gestante não for capaz de interromper o tabagismo apenas com o auxílio de intervenções não-farmacológicas, deve-se considerar o uso de TRN. Este tipo de terapia adjuvante dobra a taxa de cessação, aumentando a chance da mãe e do feto serem poupados de mais de 4700 substâncias contidas no cigarro além da nicotina, especialmente do monóxido de carbono, que os autores consideram ser o composto de maior toxicidade do tabaco. Na gravidez, as formulações de liberação intermitente (goma de mascar, por exemplo) devem ser preferidas por disponibilizarem ao feto uma dose total diária de nicotina menor do que os dispositivos de liberação lenta (adesivo). Além disso, Dempsey e Benowitz recomendam o uso de TRN para mães que precisam parar de fumar e estão amamentando, já que a quantidade de nicotina excretada no leite materno é bem pequena, trazendo um risco mínimo ao bebê, frente ao largo benefício da proteção ao tabagismo passivo. Outros autores ${ }^{80,81}$ também entendem que, apesar de a TRN não estar idealmente indicada para uso durante a gestação, a sua utilização ainda é mais segura do que continuar fumando.

Devido às limitações do emprego da farmacoterapia no controle do tabagismo durante a gestação, os aspectos psicológicos da dependência devem ser amplamente abordados por meio de psicoterapia. Variando de acordo com o grau de motivação da gestante, várias técnicas podem ser empregadas com sucesso e aplicadas individualmente ou em grupo ${ }^{79,82-84}$. Utiliza-se a entrevista motivacional especialmente para as gestantes ambivalentes e a terapia cognitivocomportamental para aquelas já motivadas, sendo esta a técnica mais aplicada nos programas de tratamento do tabagismo ${ }^{57}$, inclusive no programa proposto pelo Ministério da Saúde do Brasil ${ }^{85}$. Aconselhamento pessoal ou por telefone também mostram-se eficazes ${ }^{79,82}$. Materiais de auto-ajuda 
com orientações práticas de técnicas de cessação do tabagismo sumarizadas em livretos, fitas $\mathrm{K} 7$ e de vídeo, ou em programas de computador têm resultado limitado se utilizados isoladamente, porém podem ser úteis se inseridos no contexto de outras formas de intervenção ${ }^{59}$. Esses meios auxiliares são tão mais eficientes quanto mais forem especificamente orientados para o público-alvo ${ }^{79,82}$. Daí a importância de as ações públicas e privadas de controle do tabagismo preverem programas e materiais individualizados para as gestantes. Diferentemente do Canadá, EUA, e Inglaterra, o Brasil não tem uma política bem definida de apoio às gestantes para a interrupção do fumo na gravidez. Programas e recomendações ${ }^{42,86}$ voltados especificamente para este grupo, com informações práticas, disponíveis inclusive na web ( w w w.surgeongeneral.gov/tobacco/ p r e n a t a l. h t m, w w w. c c t c. c a, www.smokefreefamilies.uab.edu/bpi_form.htm), têm apresentado excelentes resultados e podem servir de modelo para ações regionalizadas de mesma envergadura no Brasil.

\section{Prevenção da recaída}

A cessação do tabagismo no início da gestação acarreta importante redução dos riscos à saúde fetal $^{4}$. A persistência da abstinência no pós-parto evita a exposição passiva do neo-nato ao compostos do tabaco, a qual está associada a várias doenças, principalmente às infecções respiratórias. A abstinência mantida definitivamente implicará no ganho de qualidade de vida da criança, e também na diminuição dos riscos maternos de doenças relacionadas ao tabaco. Estes aspectos dão grande relevância à preocupação com o fenômeno da recaída.

Apesar de 25\% a 40\% das fumantes pararem de fumar quando tomam ciência de que estão grávidas ${ }^{59,87,88}$, ou reduzirem drasticamente 0 consumo de cigarros $^{89}$, a recaída pré e pós-parto é muito freqüente. Estima-se que cerca de $12 \%$ a $15 \%$ das gestantes que alcançam a cessação, seja de forma espontânea ou com auxílio, vão recair ainda durante a gestação ${ }^{90}$. Após o término da gravidez, o risco é muito maior, atingindo taxas de $40 \%$ a $90 \%$ nos doze primeiros meses do pós-parto ${ }^{91,92}$.

As variáveis mais freqüentemente citadas como preditivas de recaída durante a gravidez são: pouca credibilidade sobre os malefícios do tabaco ao feto, julgamento de baixa capacidade de se manter sem fumar, multiparidade, falta de suporte social (convivência com fumantes), estresse, periodo curto de cessação antes da primeira visita prénatal, baixa escolaridade e gravidez precoce ${ }^{88,93}$.

Quinn et al. ${ }^{93}$, estudando o comportamento de gestantes da Califórnia que interromperam espontaneamente o hábito tabágico por ocasião de sua gestação, encontraram uma taxa de recaída, ainda no pré-natal, em torno de $21 \%$. Face aos achados, alertaram sobre o alto índice de recaída entre as que cessaram espontanemente e advertiram que estas devem ser identificadas e incluídas em um programa especial para prevenção de recaída, e não simplesmente parabenizadas, como costuma ocorrer.

Por outro lado, Secker-Walker et al. ${ }^{94}$ mostram que os programas de prevenção de recaída oferecidos no pré e pós-natal, tanto para as que cessaram espontaneamente como para as que cessaram com auxílio, não modificam a taxa de recaída a longo prazo no pós-parto. No entanto, prolongam o tempo de abstinência, o que justifica sua aplicação a todos os grupos de gestantes. Outros trabalhos também mostram a importância desses programas, independentemente da taxa de recaída a longo prazo ${ }^{91}$.

Em um estudo prospectivo, McBride et al. ${ }^{95}$ acompanharam 106 mulheres que haviam parado de fumar durante a gestação. Na sexta semana pós-parto, 24\% já haviam retomado o tabagismo de forma regular e ao término do sexto mês, a taxa de recaída era de 40\%. Em concordância com os achados de Fingerhut ${ }^{96}$ e Mullen ${ }^{92}$, observaram que a recaída era gradual, com o maior risco por volta do quarto mês, diferentemente do que ocorre com outros grupos, onde a recaída se dá precocemente (em dias ou semanas).

Os fatores relacionados aos riscos de recaída após o término da gestação são: cessação tardia durante a gravidez ${ }^{96}$; interrupção precoce do aleitamento materno ${ }^{97,98}$; ganho de peso ${ }^{97}$; estresse com os cuidados do bebê $\hat{e}^{99}$; cônjuge fumante ${ }^{95}$; depressão pós-parto ${ }^{60}$; término da licença maternidade, quando a mãe, além das tarefas domiciliares, volta a assumir os conflitos profissionais e a conviver com colegas fumantes no trabalho.

A prevenção e o tratamento da recaída devem ser intensamente trabalhados, pois este fenômeno pode comprometer a chance de um abandono 
definitivo do tabagismo, alcançado pela motivação relacionada à gestação.

\section{COMENTÁRIOS FINAIS}

Os malefícios do tabaco atingem não apenas a gestante, mas também o feto, o qual, ainda no útero, se torna um verdadeiro fumante ativo.

Dados recentes da $\mathrm{OMS}^{100}$ revelam que a cada ano cerca de cinco milhões de pessoas morrem em todo o mundo pela ação do tabaco. Esses dados alarmantes mostram a necessidade de controle do tabagismo.

As gestantes devem estar no centro das atenções das ações. Os programas para cessação direcionados às gestantes têm resultados muito superiores àqueles destinados à população em geral. A relação custo-benefício fica otimizada nesse grupo. Com pequenos investimentos alcança-se grandes resultados com a eliminação das exposições materna e fetal ao tabaco, além da proteção do recém-nato, da criança em desenvolvimento e dos demais parentes, do fumo passivo. A figura da mãe é fundamental na educação: seus hábitos norteiam o comportamento social dos filhos, e podem influenciar a decisão de futuras gerações em relação ao início do hábito do tabagismo.

Alguns grupos merecem considerações e estratégias especiais visando a otimizar a abordagem do fumo na gravidez: jovens em idade fértil, visto que a cessação antes da concepção é a garantia de proteção máxima ao feto, e a farmacoterapia de apoio no tratamento da dependência nicotínica pode ser utilizada sem as restrições da gestação; gestantes com baixa escolaridade, pois o maior consumo de tabaco se concentra entre estas gestantes; gestantes que cessaram espontaneamente o hábito de fumar, pela alta prevalência de recaída; cônjuges fumantes, por influenciarem negativamente na tentativa de cessação da gestante; nas gestantes que alcançaram a cessação durante a gravidez, o acompanhamento no pós-parto se torna imprescindivel para garantir a cessação definitiva, já que existe um alto risco de recaída após a gestação.

É necessário que obstetras e pediatras estejam conscientes e que enfermeiros e agentes de saúde sejam treinados em relação ao controle do tabagismo. Esses profissionais são a base do sucesso dos programas de abordagem do fumo na gravidez e primeira infância. Para tornar bem sucedida esta realidade em nosso país, é necessário desenvolver uma política bem definida de apoio à cessação do tabagismo durante a gestação. Os diversos seguimentos da sociedade, como governo, imprensa, juristas, educadores, líderes religiosos, conselhos, sociedades e entidades de classe da área de saúde devem reconhecer sua importância na melhoria da saúde da população. Nesse contexto, deve ser ressaltado que o controle do tabagismo é o melhor e mais barato meio de se prevenir, tratar e curar várias doenças.

\section{REFERÊNCIAS}

1 from the Centers for Disease Control. Health benefits of smoking cessation. JAMA 1990;264:1930.

2 Lambers DS, Clark KE. The maternal and fetal physiologic effects of nicotine. Semin Perinatol 1996;20:115-26.

3 Medical-care expenditures attributable to cigarette smoking during pregnancy - United States, 1995. MMWR Morb Mortal Wkly Rep 1997;46:1048-50.

4 Butler NR, Goldstein H, Ross EM. Cigarette smoking in pregnancy: its influence on birth weight and perinatal mortality. Br Med J 1972;2:127-30.

5 Youth tobacco surveillance - United States, 1998-1999. MMWR CDC Surveill Summ 2000;49:1-94.

6 Aligne CA, Stoddard JJ. Tobacco and children. An economic evaluation of the medical effects of parental smoking. Arch Pediatr Adolesc Med 1997;151:648-53.

7 Dempsey DA, Benowitz NL. Risks and benefits of nicotine to aid smoking cessation in pregnancy. Drug Saf 2001;24:277-322.

8 Cutler AR, Wilkerson AE, Gingras JL, Levin GD. Prenatal cocaine and/or nicotine exposure in rats: preliminary findings on long-term cognitive outcome and genital development at birth. Neurotoxicol Teratol 1996;18:635-643.

9 Schlumpf M, Gahwiler M, Ribary U, Lichtensteiger W. A new device for monitoring early motor development: prenatal nicotine-induced changes. Pharmacol Biochem Behav 1988;30:199-203.

10 Peters DA, Tang S. Sex-dependent biological changes following prenatal nicotine exposure in the rat. Pharmacol Biochem Behav 1982;17:1077-1082.

11 Slotkin TA. Fetal nicotine or cocaine exposure: which one is worse? J Pharmacol Exp Ther 1998;285:931-45.

12 Schoendorf KC, Kiely JL. Relationship of sudden infant death syndrome to maternal smoking during and after pregnancy. Pediatrics 1992;90:905-8.

13 Slotkin TA, Lappi SE, McCook EC, Lorber BA, Seidler FJ. Loss of neonatal hypoxia tolerance after prenatal nicotine exposure: implications for sudden infant death syndrome. Brain Res Bull 1995;38:69-75.

14 Gilliland FD, Berhane K, McConnell R, Gauderman WJ, Vora H, Rappaport EB, Avol E, Peters JM. Maternal smoking during pregnancy, environmental tobacco smoke exposure and childhood lung function. Thorax 2000;55:271-6.

15 Gilliland FD, Li YF, Peters JM. Effects of maternal smoking during pregnancy and environmental tobacco smoke on asthma and wheezing in children. Am J Respir Crit Care Med 2001;163:429-36. 
16 Elliot JG, Carroll NG, James AL, Robinson PJ. Airway alveolar attachment points and exposure to cigarette smoke in utero. Am J Respir Crit Care Med 2003; $167: 45-9$.

17 Sherrill DL, Lebowitz MD, Knudson RJ, Burrows B. Smoking and symptom effects on the curves of lung function growth and decline. Am Rev Respir Dis 1991;144:17-22.

18 Tager IB, Weiss ST, Munoz A, Rosner B, Speizer FE. Longitudinal study of the effects of maternal smoking on pulmonary function in children. N Engl J Med 1983;309:699-703.

19 Newnham JP, Patterson L, James 1, Reid SE. Effects of maternal cigarette smoking on ultrasonic measurements of fetal growth and on doppler flow velocity waveforms. Early Hum Dev 1990;24:23-36.

20 Jarvis M, Tunstall-Pedoe H, Feyerabend C, Vesey C, Salloojee Y. Biochemical markers of smoke absorption and self-reported exposure to passive smoking. J Epidemiol Community Health 1984;38:335-9.

21 Sauter D. Hematologig principles. In: Goldfrank LR, Weisman RS, Flomenbaum NE, et al., editors. Toxicologic emergencies. Norwalk: Appleton \&t Lange; 1994.

22 Crowley TJ, Andrews AE, Cheney J, Zerbe G, Petty TL. Carbon monoxide assessment of smoking in chronic obstructive pulmonary disease. Addict Behav 1989;14:493-502.

23 Longo LD. The biological effects of carbon monoxide on the pregnant woman, fetus, and newborn infant. Am J Obstet Gynecol 1977;129:69-103.

24 Gabriel R, Alsat E, Evain-Brion D. Alteration of epidermal growth factor receptor in placental membranes of smokers: relationship with intrauterine growth retardation. Am J Obstet Gynecol 1994;170:1238-43.

25 Buchan PC. Cigarette smoking in pregnancy and feta hyperviscosity. Br Med J (Clin Res Ed) 1983;286:1315.

26 Bureau MA, Shapcott D, Berthiaume Y, Monette J, Blouin D, Blanchard P, et al. Maternal cigarette smoking and fetal oxygen transport: a study of P50, 2,3Diphosphoglycerate, total hemoglobin, hematocrit, and type $f$ hemoglobin in fetal blood. Pediatrics 1983;72:22-6.

27 Penney DG. Effects of carbon monoxide exposure on developing animals and humans. In: Penney DG, editor. Carbon monoxide. Boca Raton: CRC Press; 1996. p.10944.

28 Clubb FJ, Jr., Penney DG, Baylerian MS, Bishop SP. Cardiomegaly due to myocyte hyperplasia in perinatal rats exposed To 200 Ppm carbon monoxide. J Mol Cell Cardiol 1986;18:477-86.

29 Schellenberg JC, North RA, Taylor R, Zhou RL. Secretory component of immunoglobulin $A$ in maternal serum and the prediction of preterm delivery. Am J Obstet Gynecol 1998; 178:535-9.

30 Meyer MB, Tonascia JA. Maternal smoking, pregnancy complications, and perinatal mortality. Am J Obstet Gynecol 1977;128:494-502.

31 Hadley CB, Main DM, Gabbe SG. Risk factors for preterm premature rupture of the fetal membranes. Am J Perinato 1990;7:374-9.

32 Barrett B, Gunter E, Jenkins J, Wang M. Ascorbic acid concentration in amniotic fluid in late pregnancy. Bio Neonate $1991 ; 60: 333-5$.
33 Anderson R. Effects of ascorbate on leucocytes: part 11. Effects of ascorbic acid and calcium and sodium ascorbate on neutrophil phagocytosis and post-phagocytic metabolic activity. S Afr Med J 1979;56:401-4.

34 Phillips CL, Tajima S, Pinnell SR. Ascobic acid and transforming growth factor-B-1 increase collagen biosynthesis via different mechanismis: coordinate regulation of pro-alpha-1(1) and pro-alpha-1(111) collagens. Arch Biochem Biophys 1982;295:397-403.

35 Sastry BV. Placental toxicology: tobacco smoke, abused drugs, multiple chemical interactions, and placental function. Reprod Fertil Dev 1991;3:355-72.

36 French Jl, Mcgregor JA. The pathobiology of premature rupture of membranes. Semin Perinatol 1996;20:34468.

37 Keelan JA, Coleman M, Mitchell MD. The molecular mechanisms of term and preterm labor: recent progress and clinical implications. Clin Obstet Gynecol 1997;40:460-78.

38 Klerman LV, Rooks JP. A simple, effective method that midwives can use to help pregnant women stop smoking. J Nurs Midwifery 1999;44:118-23.

39 Murray RP, Connett JE, Lauger GG, Voelker HT. Error in smoking measures: effects of intervention on relations of cotinine and carbon monoxide to self-reported smoking. The Lung Health Study Research Group. Am J Public Health 1993;83:1251-7.

40 Glasgow RE, Mullooly JP, Vogt TM. Biochemical validation of smoking status: pros, cons, and data from four lowIntensity interventio. Addict Behav 1993;18:511-27.

41 Velicer WF, Prochaska J0, Rossi JS, Snow MG. Assessing outcome in smoking cessation studies. Psychol Bull 1992;111:23-41.

42 Windsor RA, Woodby LL, Miller TM, Hardin JM, Crawford MA, DiClemente CC. Effectiveness of agency for health care policy and research clinical practice guideline and patient education methods for pregnant smokers in medicaid maternity care. Am J Obstet Gynecol 2000;182:68-75.

43 Secker-Walker RH, Vacek PM, Flynn BS, Et Al. Exhaled carbon monoxide and urinary cotinine as measures of smoking In pregnancy. Addict Behav 1997;22:671-84.

44 Laranjeira R, Pillon SC, Dunn J. Environmental tobacco smoke exposure among non-smoking waiters: measurement of expired carbon monoxide levels. Rev Paul Med 2000;118:89-92.

45 Adams EK, Melvin CL. Costs of maternal conditions attributable to smoking during pregnancy. Am J Prev Med 1998;15:212-9.

46 Ronald F, Cobun MD. Endogenous carbon monoxide production. N Engl J Med 1970;282:207-9.

47 Halpern R, Schaefer ES, Pereira A da S, Arnt EM, Bezerra JP, Pinto dos S. Risk factors for low birth weight in a rural community in southern Brazil. J Pediatr 1996;72:369-73.

48 WHO. Division of Family Planning. The incidence of low birth weight. A critical review of available information. World Health Stat Q 1980;33:197-224.

49 Difranza JR, Lew RA. Effect of maternal cigarette smoking on pregnancy complications and sudden infant death Syndrome. J Fam Pract 1995;40:385-94.

50 Marks JS, Koplan JP, Hogue CJ, Dalmant ME. A costbenefit/cost-effectiveness analysis of smoking cessation for pregnant women. Am J Prev Med 1990;6:282-9. 
51 Adams EK, Miller VP, Ernst C, Nishimura BK, Melvin C, Merritt R. Neonatal health care costs related to smoking during pregnancy. Health Econ 2002;11:193-206.

52 Difranza JR, Lew RA. Morbidity and mortality in children associated with the use of tobacco products by other people. Pediatrics 1996;97:560-8.

53 Doubilet P, Weinstein MC, McNeil BJ. Use and misuse of the term "cost effective" in medicine. N Engl J Med 1986;314:253-6.

54 Ravenholt RT. Tobacco's impact on twentieth-century u.s. mortality patterns. Am J Prev Med 1985;1:4-17.

55 The World Bank. Curbing the epidemic: governments and the economics of tobacco control. Washington: The World Bank; 1999. Series: Development in practice.

56 Secker-Walker RH, Vacek PM, Flynn BS, Mead PB. Estimated gains in birth weight associated with reductions in smoking during pregnancy. J Reprod Med 1998;43:96774.

57 Rigotti NA. Clinical practice. Treatment of tobacco use and dependence. N Engl J Med 2002;346:506-12.

58 Melvin CL, Dolan-Mullen P, Windsor RA, Whiteside HP Jr, Goldenberg RL. Recommended cessation counselling for pregnant women who smoke: a review of the evidence. Tob Control 2000;9(Suppl 3):11180-84.

59 Windsor RA, Lowe JB, Perkins LL, Smith-Yoder D, Artz L, Crawford M, et al. Health education for pregnant smokers: its behavioral impact and cost benefit. Am J Public Health 1993;83:201-6.

60 Orleans CT, Barker DC, Kaufman NJ, Marx JF. Helping pregnant smokers quit: meeting the challenge in the next Decade. Tob Control 2000;9(Suppl 3):1116-11.

61 Connor SK, Mcintyre L. The sociodemographic predictors of smoking cessation among pregnant women in Canada. Can J Public Health 1999;90:352-5.

62 US Dept of Health and Human Services. Healthy people 2000 review. Washington, DC: Office of Disease Prevention and Health Promotion; 1998-1999. DHHS Publication (PHS) 99-1256.

63 Pickett KE, Abrams B, Schauffler HH, Savage J, Brandt P, Kalkbrenner A, et al. Coverage of tobacco dependence treatments for pregnant smokers in health maintenance organizations. Am J Public Health 2001;91:1393-4.

64 Mcphillips-Tangum C. Results from the first annual survey on addressing tobacco in managed care. Tob Control 1998;7(Suppl):S11-13

65 Raw M, Mcneill A, West R. Smoking cessation guidelines for health professionals. A guide to effective smoking cessation interventions for the health care system. Health education authority. Thorax 1998;53(Suppl 5 Pt 1):S119.

66 SAMHSA 1997. Office of Applied Studies, Substance Abuse and Mental Health Services Administration. Preliminary results from the 1996 national household survey on drug abuse. Rockville, MD: SAMHSA; 1997. p.90:Table 32B. DHHS Publication (SMA) 97-3149.

67 Ebrahim SH, Floyd RL, Merritt RK 2nd, Decoufle P, Holtzman D. Trends in pregnancy-related smoking rates in the United States, 1987-1996. JAMA 2000;283:3616.

68 Horta BL, Victora CG, Barros FC, dos Santos 1 da S, Menezes AM. Tobacco smoking among pregnant women in an urban area in southern Brazil, 1982-93. Rev Saude Publica 1997;31:247-53.
69 Gross R, Mauad Filho F, Netto AR, Sobrinho FM, Ferreira DL, Mucallo G, et al. Smoking and pregnancy. l. Prevalence of the smoking habit among pregnant women. Rev Assoc Med Bras 1983;29:4-6.

70 Simoes MJ. Study of yhe frequency of mothers' smoking habits during pregnancy in Ribeirão Preto - SP. Rev Cienc Biomed 1985;6:61-9.

71 Halal IS, Victoria CG, Barros FC. Determining factors related to smoking and its abandonment during pregnancy in an Urban locality in southern Brazil. Rev Saude Publica 1993;27:105-12.

72 Albrecht S, Cassidy B, Reynolds MD, Ketchem S, Abriola D. Developing guidelines for smoking cessation interventions for pregnant adolescents. J Pediatr Nurs 1999;14:150-6.

73 Fiore MC. The new vital sign. assessing and documenting smoking status. JAMA 1991;266:3183-4.

74 Laml T, Hartmann BW, Kirchengast S, Preyer 0, Albrecht AE, Husslein PW. Impact of maternal anthropometry and smoking on neonatal birth weight. Gynecol Obstet Invest 2000;50:231-6.

75 Diclemente CC, Dolan-Mullen P, Windsor RA. The process of pregnancy smoking cessation: implications for interventions. Tob Control 2000;9(Suppl 3):11116-21.

76 Wenderlein JM. Smoking and pregnancy. Z Arztl Fortbild (Jena) 1995;89:467-71.

77 Malbergier A. Tabagismo. J Bras Dep Quim 2001;2:47-51.

78 Brasil. Ministério da Saúde. Programa Nacional de Controle do Tabagismo. Fumo. Portaria 1575/GM do MS. DOU 170, 03 set. 2002.

79 Fiore MC, Bailey WC, Cohen SJ, et al. Treating tobacco use and dependence. Rockville, MD: Department of Health and Human Services, Public Health Service; 2000.

80 Gigliotti A, Laranjeira R. Terapêutica do tabagismo. J Bras Med 1999;77:66-78.

81 WHO. Women and the tobacco epidemic: challenges for the 21 st century. Geneva; 2001.

82 Lancaster T, Stead L, Silagy C, Sowden A. Effectiveness of interventions to help people stop smoking: findings from the Cochrane Library. BMJ 2000;321:355-8.

83 Miller WR, Rollnick S. Miller WR, Rollnick S. Motivational interviewing: preparing people for change. New York: Guiford Press; 1991.

84 Leite JC, Franken RA. Group psychotherapy in the treatment of nicotine dependence: the experience at Santa Casa of São Paulo. Rev Soc Cardiol Estado São Paulo 1999;6(Suppl A):1-10.

85 INCA - Instituto Nacional do Câncer. Abordagem e tratamento do fumante - consenso 2001. Rio de Janeiro: INCA; 2001.

86 Walsh RA, Redman S, Brinsmead MW, Byrne JM, Melmeth A. A smoking cessation program at a public antenatal clinic. Am J Public Health 1997;87:1201-4.

87 Ershoff DH, Mullen PD, Quinn VP. A randomized trial of a serialized self-help smoking cessation program for pregnant women in an HMO. Am J Public Health 1989;79:182-7.

88 Valbo A, Schioldborg P. Smoking in pregnancy: a followup study of women unwilling to quit. Addict Behav 1993;18:253-7.

89 Floyd RL, Rimer BK, Giovino GA, Mullen PD, Sullivan SE. A review of smoking in pregnancy: effects on pregnancy outcomes and cessation efforts. Annu Rev Public Health 1993; 14:379-411. 
90 Petersen L, Handel J, Kotch J, Podedwomy T, Rosen A. Smoking reduction during pregnancy by a program of selfhelp and clinical support. Obstet Gynecol 1992;79:924-30.

91 Mcbride CM, Curry SJ, Lando HA, Pirie PL, Grothaus LC, Nelson JC. Prevention of relapse in women who quit smoking during pregnancy. Am J Public Health 1999;89:706-11.

92 Mullen PD, Quinn VP, Ershoff DH. Maintenance of nonsmoking postpartum by women who stopped smoking during pregnancy. Am J Public Health 1990;80:992-4.

93 Quinn VP, Mullen PD, Ershoff DH. Women who stop smoking spontaneously prior to prenatal care and predictors of relapse before delivery. Addict Behav 1991;16:29-40.

94 Secker-Walker RH, Solomon LJ, Flynn BS, Skelly JM, Lepage SS, Goodwin GD, et al. Smoking relapse prevention counseling during prenatal and early postnatal care. Am J Prev Med 1995;11:86-93.
95 Mcbride CM, Pirie PL, Curry SJ. Postpartum relapse to smoking: a prospective study. Health Educ Res 1992;7:381-90.

96 Fingerhut LA, Kleinman JC, Kendrick JS. Smoking before, during, and after pregnancy. Am J Public Health 1990;80:541-44.

97 Mcbride CM, Pirie PL. Postpartum smoking relapse. Addict Behav 1990;15:165-68.

98 O'Campo P, Faden RR, Brown H, Gielen AC. The impact of pregnancy on women's prenatal and postpartum smoking behavior. Am J Prev Med 1992;8:8-13.

99 Edwards N, Sims-Jones N. Smoking and smoking relapse during pregnancy and postpartum: results of a qualitative study. Birth 1998;25:94-100.

100 WHO. The tobacco atlas. Geneva; 2002. 